Note

\title{
Gut microbial composition of elderly women born in the Japanese longevity village Ogimi
}

\author{
Hiroto MORITA ${ }^{1 *}$, Mutsuki ICHISHIMA ${ }^{1}$, Ipputa TADA ${ }^{2,3}$, Hirotsugu SHIROMA ${ }^{2,4}$, Makoto MIYAGI ${ }^{2}$, \\ Teppei NAKAMURA ${ }^{1}$, Hiroshi TANAKA ${ }^{2}$ and Shinya IKEMATSU ${ }^{2}$ \\ ${ }^{1}$ Department of Microbiological Flora Technology, Core Technology Laboratories, Asahi Quality \& Innovations, Ltd., 11-10-5 Fuchinobe, \\ Sagamihara, Kanagawa 252-0206, Japan \\ ${ }^{2}$ Department of Bioresources Engineering, National Institute of Technology, Okinawa College, 905 Henoko, Nago, Okinawa 905-2192, \\ Japan \\ ${ }^{3}$ Present address: Department of Genetics, The Graduate University for Advanced Studies, SOKENDAI, 1111 Yata, Mishima, \\ Shizuoka 411-8540, Japan \\ ${ }^{4}$ Present address: School of Life Science and Technology, Tokyo Institute of Technology, 2-12-1 Ookayama, Meguro-ku, \\ Tokyo 152-8550, Japan
}

Received January 7, 2020; Accepted July 28, 2020; Published online in J-STAGE August 21, 2020

\begin{abstract}
Ogimi is one of Japan's longevity villages and is located in Okinawa Prefecture. In this study, we focused on the elderly women living in the village, classified them into two groups based on whether or not they lived in Ogimi during the first 3 years of their lives, and compared the gut microbiota between the two groups. There were no differences in alpha and beta diversity; however, we found that the elderly women who lived in Ogimi during the first 3 years of their lives had a higher rate of Akkermansia muciniphila colonization in their guts.
\end{abstract}

Key words: microbiota, Akkermansia muciniphila, longevity, early-life environment

It is believed that human health is determined by the complex interactions between different factors, including genetics, epigenetics, diet, activity level, and lifestyle. In recent years, many studies have shown that the human microbial community formed in the gastrointestinal tract is another factor involved in our health. The formation of the gut microbiota begins immediately after birth, and the delivery method (i.e., vaginal delivery or cesarean section) and food source in infancy (formula or breast milk) influence the initial composition of the microbiota [1-4]. An adult-like gut microbiota is established in the first 3 to 5 years of life, and it remains stable throughout a person's lifetime, but it may change depending on both environmental and host factors, such as lifestyle, diet, antibiotics, genetics, gut senescence, and immune senescence [5-7]. Thus, the composition and relative abundance of different bacterial taxa in the human adult gut microbiota differ from country to country and between the elderly and young adults [8-10].

Okinawa Prefecture is one of the 47 prefectures of Japan and is located in the southernmost part of the country. Since the prefecture was an independent country before 1875 , called the Ryukyu Kingdom, its unique cultural lifestyle and food still remain, especially in the northern area of the main island of Okinawa.
Furthermore, it has been reported that the genetic background differs between the Ryukyu people (called Ryukyuans), who live mainly in Okinawa, and the Japanese people living on the mainland of Japan [11]. Ogimi is a village located in the northern region of Okinawa's main island and has a population of approximately 3,000 . The village is known as a village of longevity in Japan, and thus the lifestyle and foods of the elderly living in the village have been researched $[12,13]$. However, the gut microbiota of the residents has not yet been researched. According to data published by the Ministry of Health, Labour and Welfare (https:// www.mhlw.go.jp/toukei/saikin/hw/life/ckts15/index.html), the average lifespan of women living in the village is longer than the average Japanese woman's lifespan, but that of the men living in the village is shorter than the average Japanese man's lifespan; thus, we focused on the elderly women living in Ogimi and investigated the profile of the gut microbiota in them. The study protocol was approved by the Ethical Committee for Research in Humans of Shiba Palace Clinic (Tokyo, Japan) in compliance with the Declaration of Helsinki (permission No. ASHB rn20876). Informed consent was provided by all participants prior to enrollment. In total, 60 Japanese women who were over 70 years of age and living in Ogimi as of August 2016 were enrolled in this

*Corresponding author. Hiroto Morita (E-mail: hiroto.morita@asahi-qi.co.jp)

(Supplementary materials: refer to PMC https://www.ncbi.nlm.nih.gov/pmc/journals/2480/)

(C)2021 BMFH Press

This is an open-access article distributed under the terms of the Creative Commons Attribution Non-Commercial No Derivatives (by-nc-nd) License. (CC-BY-NC-ND 4.0: https://creativecommons.org/licenses/by-nc-nd/4.0/) 


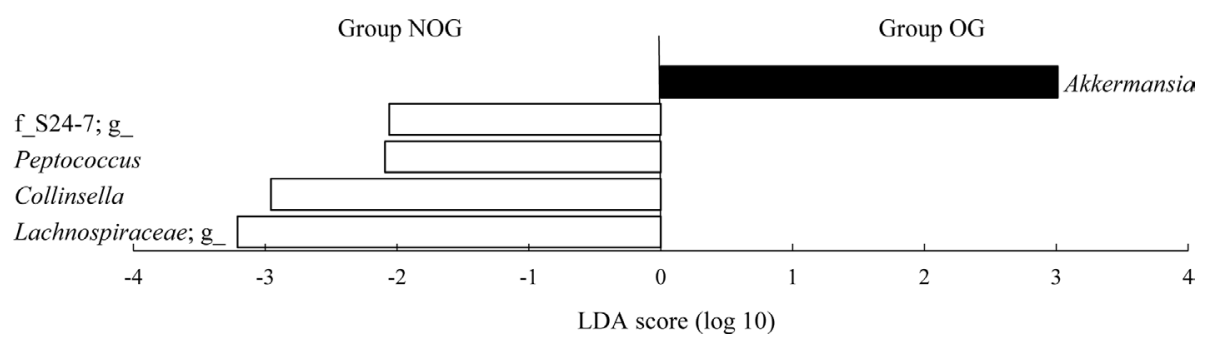

Fig. 1. Most likely bacterial genera to explain differences between groups.

Three known and two unidentified genera were detected by the LEfSe analytic method (log-linear discriminant analysis [LDA] score $>2$ ). Bacterial taxa significantly enriched in group OG is shown on the right side, and those in group NOG are shown on the left side.

study. Since the human gut microbiota is established during the first 3 to 5 years of life, we classified the women into two groups based on whether they were living in Ogimi during the first 3 years of their lives (group OG, $\mathrm{n}=40$ ) or not (group NOG, $\mathrm{n}=20$ ) and compared the gut microbiotas between the two groups. The areas where they lived from their birth to the present are shown in Supplementary Table 1 . The mean age \pm standard deviation (SD) of group OG was $83.8 \pm 6.8$ years, and that of group NOG was $81.3 \pm 6.1$ years $(\mathrm{p}=0.16$, student's t-test). The gut microbiota of all participants were characterized by Illumina MiSeq sequencing of the V4 region of the bacterial 16S rRNA gene using DNA extracted from fecal samples. These analyses were carried out as previously described [14]. In total, 3,558,508 high-quality filtered sequences were obtained, and we first compared the Chao1, Shannon diversity index, and phylogenetic distance (PD) whole tree between groups OG and NOG. All data were expressed as the mean $\pm \mathrm{SD}$, and p-values were calculated by the two-sample t-test using Monte Carlo permutations. The results revealed no significant differences (Chao1, 1,468 \pm 495 for group OG and $1,563 \pm 452$ for group NOG, $\mathrm{p}=0.62$; Shannon diversity index, $5.81 \pm 1.00$ for group OG and $5.99 \pm 0.97$ for group NOG, $\mathrm{p}=0.50$; PD whole tree, $38.68 \pm 11.94$ for group OG and $40.39 \pm 11.64$ for group NOG, $p=0.62$ ). Then, principal coordinate analysis based on the unweighted or weighted UniFrac distance was performed, and no significant differences were observed between the two groups ( $\mathrm{p}=0.36$ and 0.39 , respectively, with $\mathrm{p}$-values determined by permutational multivariate analysis of variance). These results showed that there were no differences between groups OG and NOG in the overall gut microbiota. In studies which focused on the gut microbiota of centenarians or elderly people living in longevity areas, differences were observed in the diversity of the gut microbiota, and this conflicts with our results [15-18]. The previous studies grouped subjects according to age and/or living area, which may have contributed to the differences found in their gut microbiotas. However, our study compared two groups that differed in terms of their early-life environments but matched in terms of age, gender, and current living area. This might explain why no differences were observed in our microbial diversity analysis.

The microbial compositions of both groups at the genus level and adjusted p-values of the Mann-Whitney $U$ test with Benjamini-Hochberg false discovery rate correction (q-value) are shown in Table 1. No differences were observed in the relative abundances of each genus between two groups if q-value $<0.05$ was considered to indicate statistical significance. However, when our microbiota data were analyzed with the linear discriminant analysis effect size algorithm (LEfSe) (http://huttenhower.sph. harvard.edu/galaxy/), 5 genera were found to be significantly different between the groups (Fig. 1). Akkermansia was more abundant in group OG, and Lachnospiraceae;g, Collinsella, Peptococcus, and f S24 7;g were more abundant in group NOG. Akkermansia muciniphila, which was isolated from human feces in 2004 [19], is expected to be a next-generation probiotic. The administration of live $A$. muciniphila partially protected against high-fat diet-induced obesity and insulin resistance in mice [20], and oral administration of pasteurized A. muciniphila significantly improved insulin sensitivity and reduced plasma total cholesterol in humans [21]. To determine the cell counts and detection rate of $A$. muciniphila in groups $\mathrm{OG}$ and NOG, we calculated the cell counts of $A$. muciniphila in fecal samples by quantitative PCR using A. muciniphila-specific primers [22]. Quantitative PCR amplification and detection were performed on a LightCycler 480 Instrument II (Roche Diagnostics K.K., Tokyo, Japan) using SYBR Premix Ex Taq II (Takara Bio Inc., Kusatsu, Japan). The standard curve was generated using a synthesized partial $16 \mathrm{~S}$ rRNA gene of $A$. muciniphila and serial 10-fold diluents. The partial 16S rRNA gene was a 429 bp fragment that contained the region from the forward primer annealing position to the reverse primer annealing position (329 bp) and $50 \mathrm{bp}$ up- and downstream of that region. The fragment was synthesized by Thermo Fisher Scientific K.K. (Tokyo, Japan). Due to A. muciniphila having 3 copies of the 16S rRNA gene, copy numbers measured by the quantitative PCR were divided by 3 to calculate the cell count of A. muciniphila. A. muciniphila was detected in 27 of 40 group OG fecal samples and in 8 of 20 group NOG fecal samples. The detection rate of $A$. muciniphila in group OG was significantly higher than that in group NOG (Table 2). Also, the cell count of $A$. muciniphila was higher in group OG than in group NOG (Table 2). However, when the cell counts of $A$. muciniphila were compared using only the data from participants in which $A$. muciniphila was detected, no significant difference was observed between the groups (Table 2). These results indicated that the elderly women who lived in Ogimi during the first 3 years of their lives had a higher rate of $A$. muciniphila colonization in their guts than those who were born in other regions and moved to Ogimi.

In the Japanese elderly, the relative abundance of Akkermansia has been reported to be in the range of 0 to $0.79 \%$ [23]. The relative abundance of this bacteria in the group OG ranged from 0 to $13.6 \%$, and the mean was $1.2 \%$. For the group NOG, relative abundance ranged from 0 to $2.3 \%$, and the mean was $0.2 \%$. Due to differences in methodology of microbiota analysis, 
Table 1. Relative abundance of genera in feces from groups OG and NOG

\begin{tabular}{|c|c|c|c|}
\hline \multirow{2}{*}{$\operatorname{Taxa}^{\mathrm{a})}$} & \multicolumn{2}{|c|}{ Relative abundance (mean $\pm \mathrm{SD}, \%$ ) } & \multirow{2}{*}{$\mathrm{q}$-value ${ }^{\mathrm{b})}$} \\
\hline & Group OG & Group NOG & \\
\hline f Lachnospiraceae;g Blautia & $14.9 \pm 11.8$ & $18.3 \pm 15.1$ & 0.91 \\
\hline f Streptococcaceae;g Streptococcus & $13.9 \pm 13.4$ & $8.3 \pm 9.4$ & 0.73 \\
\hline f Bifidobacteriaceae;g Bifidobacterium & $9.6 \pm 12.4$ & $9.7 \pm 12.2$ & 0.94 \\
\hline f Ruminococcaceae;g Faecalibacterium & $8.4 \pm 7.4$ & $9.6 \pm 9.4$ & 0.94 \\
\hline f_Lactobacillaceae;g_Lactobacillus & $7.4 \pm 13.7$ & $5.7 \pm 13.2$ & 0.91 \\
\hline f_Lachnospiraceae;g_Coprococcus & $6.9 \pm 5.8$ & $7.7 \pm 5.7$ & 0.91 \\
\hline f Enterobacteriaceae;g Escherichia & $4.1 \pm 10.1$ & $1.1 \pm 2.7$ & 0.84 \\
\hline f Lachnospiraceae;g [Ruminococcus] & $3.0 \pm 2.5$ & $3.4 \pm 2.4$ & 0.87 \\
\hline f_Ruminococcaceae;g_Ruminococcus & $2.4 \pm 2.7$ & $3.0 \pm 3.4$ & 0.94 \\
\hline f Enterobacteriaceae; Other & $2.3 \pm 4.6$ & $3.1 \pm 4.8$ & 0.91 \\
\hline f Lachnospiraceae;g & $2.0 \pm 2.3$ & $3.5 \pm 2.6$ & 0.21 \\
\hline f Coriobacteriaceae;g Collinsella & $1.8 \pm 2.6$ & $2.5 \pm 2.0$ & 0.29 \\
\hline f Lachnospiraceae;g Dorea & $1.7 \pm 1.7$ & $1.9 \pm 1.3$ & 0.84 \\
\hline f_Erysipelotrichaceae;g_[Eubacterium] & $1.7 \pm 3.4$ & $2.5 \pm 3.4$ & 0.73 \\
\hline o Clostridiales;f ;g & $1.5 \pm 3.2$ & $2.3 \pm 4.9$ & 0.91 \\
\hline f Lachnospiraceae;g Roseburia & $1.4 \pm 2.0$ & $1.6 \pm 2.1$ & 0.84 \\
\hline f Bacteroidaceae;g Bacteroides & $1.4 \pm 3.0$ & $0.7 \pm 0.7$ & 0.94 \\
\hline $\mathrm{f}$ Clostridiaceae;g_ SMB53 & $1.3 \pm 2.2$ & $1.2 \pm 1.8$ & 0.91 \\
\hline f_Clostridiaceae;g_Clostridium & $1.3 \pm 2.6$ & $0.9 \pm 0.9$ & 0.91 \\
\hline f Verrucomicrobiaceae;g Akkermansia & $1.2 \pm 2.7$ & $0.2 \pm 0.5$ & 0.21 \\
\hline f Lachnospiraceae;Other & $1.0 \pm 1.2$ & $1.0 \pm 0.9$ & 0.91 \\
\hline f__Ruminococcaceae;g_oscillospira & $0.8 \pm 1.0$ & $1.0 \pm 1.2$ & 0.98 \\
\hline $\mathrm{f}$ Ruminococcaceae;g & $0.8 \pm 1.2$ & $0.9 \pm 0.9$ & 0.84 \\
\hline f Methanobacteriaceae;g Methanobrevibacter & $0.7 \pm 2.2$ & $0.5 \pm 1.1$ & 0.84 \\
\hline f Erysipelotrichaceae;g & $0.6 \pm 1.0$ & $1.1 \pm 2.1$ & 0.84 \\
\hline f Enterococcaceae;g Enterococcus & $0.5 \pm 1.5$ & $0.2 \pm 0.4$ & 0.94 \\
\hline f_Ruminococcaceae; $\overline{\text { Other }}$ & $0.5 \pm 0.5$ & $0.4 \pm 0.3$ & 1.00 \\
\hline f_Erysipelotrichaceae;g_Catenibacterium & $0.5 \pm 1.4$ & $0.6 \pm 1.4$ & 0.91 \\
\hline f Leuconostocaceae;g & $0.5 \pm 1.6$ & $0.7 \pm 2.1$ & 0.85 \\
\hline f Coriobacteriaceae;g & $0.4 \pm 0.5$ & $0.6 \pm 0.5$ & 0.53 \\
\hline o Clostridiales; Other; Other & $0.4 \pm 1.0$ & $0.4 \pm 0.7$ & 0.91 \\
\hline f_Ruminococcaceae;g_Butyricicoccus & $0.3 \pm 0.7$ & $0.5 \pm 0.5$ & 0.53 \\
\hline o_ RF39;f_;g & $0.3 \pm 3.2$ & $0.4 \pm 4.9$ & 0.91 \\
\hline f [Mogibacteriaceae];g & $0.3 \pm 0.3$ & $0.3 \pm 0.3$ & 0.94 \\
\hline f Lachnospiraceae;g Anaerostipes & $0.3 \pm 0.5$ & $0.2 \pm 0.4$ & 1.00 \\
\hline f_Lachnospiraceae;g_Clostridium & $0.2 \pm 0.6$ & $0.1 \pm 0.3$ & 0.73 \\
\hline f Turicibacteraceae;g Turicibacter & $0.2 \pm 0.6$ & $0.1 \pm 0.2$ & 0.91 \\
\hline f [Mogibacteriaceae];g Mogibacterium & $0.2 \pm 0.7$ & $0.2 \pm 0.5$ & 0.91 \\
\hline f Rikenellaceae;g & $0.2 \pm 0.4$ & $0.1 \pm 0.1$ & 1.00 \\
\hline f Porphyromonadaceae;g Parabacteroides & $0.2 \pm 0.5$ & $0.1 \pm 0.1$ & 0.91 \\
\hline f_Peptostreptococcaceae;g_Clostridium & $0.2 \pm 0.7$ & $0.6 \pm 2.6$ & 0.94 \\
\hline f Prevotellaceae;g Prevotella & $0.1 \pm 0.6$ & $0.1 \pm 0.2$ & 0.53 \\
\hline f Erysipelotrichaceae;g Bulleidia & $0.1 \pm 0.8$ & $0.0 \pm 0.1$ & 0.73 \\
\hline f Christensenellaceae;g & $0.1 \pm 0.3$ & $0.1 \pm 0.2$ & 0.91 \\
\hline f_Veillonellaceae;g_Dialister & $0.1 \pm 0.5$ & $0.0 \pm 0.0$ & 0.97 \\
\hline f_Veillonellaceae;g_Megamonas & $0.1 \pm 0.7$ & $0.0 \pm 0.1$ & 1.00 \\
\hline f_Veillonellaceae;g_Veillonella & $0.1 \pm 0.4$ & $0.0 \pm 0.1$ & 0.80 \\
\hline f Methanobacteriaceae;g Methanosphaera & $0.1 \pm 0.4$ & $0.1 \pm 0.4$ & 0.84 \\
\hline f Lachnospiraceae;g Lachnospira & $0.1 \pm 0.3$ & $0.3 \pm 1.0$ & 0.84 \\
\hline f_Coriobacteriaceae;g_Eggerthella & $0.1 \pm 0.2$ & $0.1 \pm 0.2$ & 0.85 \\
\hline f Enterobacteriaceae;g & $0.1 \pm 0.2$ & $0.0 \pm 0.1$ & 0.84 \\
\hline f Erysipelotrichaceae;g Clostridium & $0.1 \pm 0.2$ & $0.1 \pm 0.2$ & 0.84 \\
\hline f Desulfovibrionaceae;g Desulfovibrio & $0.1 \pm 0.2$ & $0.0 \pm 0.1$ & 0.84 \\
\hline f Coriobacteriaceae;g Adlercreutzia & $0.1 \pm 0.1$ & $0.1 \pm 0.1$ & 0.84 \\
\hline f_Enterobacteriaceae;g_Citrobacter & $0.1 \pm 0.1$ & $0.1 \pm 0.3$ & 0.84 \\
\hline f Coriobacteriaceae; Other & $0.1 \pm 0.2$ & $0.0 \pm 0.0$ & 0.84 \\
\hline o Lactobacillales;Other;Other & $0.1 \pm 1.0$ & $0.0 \pm 0.7$ & 0.91 \\
\hline f Veillonellaceae;g Megasphaera & $0.1 \pm 0.2$ & $0.0 \pm 0.2$ & 1.00 \\
\hline f_Leuconostocaceae;g_Leuconostoc & $0.1 \pm 0.2$ & $0.1 \pm 0.2$ & 0.91 \\
\hline f Streptococcaceae;g_Lactococcus & $0.1 \pm 0.1$ & $0.3 \pm 0.8$ & 0.84 \\
\hline f Erysipelotrichaceae;g Coprobacillus & $0.1 \pm 0.1$ & $0.0 \pm 0.0$ & 0.98 \\
\hline f Clostridiaceae;g & $0.0 \pm 0.0$ & $0.1 \pm 0.4$ & 0.84 \\
\hline f_Peptococcaceae;g_Peptococcus & $0.0 \pm 0.0$ & $0.1 \pm 0.3$ & 0.21 \\
\hline
\end{tabular}

a) One hundred five genera with mean relative abundances over $0.01 \%$ in at least one group were compared. The table shows 63 genera having mean relative abundances of over $0.1 \%$ in at least one group.

b) q-values indicate adjusted p-values of the Mann-Whitney $U$ test with Benjamini-Hochberg false discovery rate correction. 
Table 2. Number of samples in which A. muciniphila was detected by qPCR and cell counts of A. muciniphila in groups OG and NOG

\begin{tabular}{lccccc}
\hline \multicolumn{1}{c}{ Participant } & Sample group & $\begin{array}{c}\text { No. of detected samples/ } \\
\text { total no. of samples (\%) }\end{array}$ & $\chi^{2}$ test $^{\text {a) }}$ & $\begin{array}{c}\text { Log no. of cells } \\
(\text { mean } \pm \text { SD)/g feces }\end{array}$ & $\begin{array}{c}\text { Mann-Whitney } \\
U \text { test }^{\text {a) }}\end{array}$ \\
\hline All participants & OG & $27 / 40(67.5)$ & 0.042 & $5.33 \pm 4.03$ & 0.040 \\
& NOG & $8 / 20(40.0)$ & & $2.97 \pm 3.89$ & $7.90 \pm 1.81$ \\
A. muciniphila detected & OG $(\mathrm{n}=27)$ & - & - & $7.43 \pm 1.77$ & 0.550 \\
participants & NOG $(\mathrm{n}=8)$ & - & & \\
\hline
\end{tabular}

a) p-values are shown.

such as differences in sample processing, DNA extraction, and subsequent processing, it is difficult to compare previously reported results with our results. However, elderly women who lived in Ogimi during the first 3 years of their lives might have more Akkermansia in their guts than Japanese elderly living in other locations.

A recent study showed that the composition of the gut microbiota is different between people living in the same city due to differences in ethnic background affecting their genetics, cultural habits, and early-life environments [24]. We did not investigate the genetic background of our participants; however, their family names indicated that more than $90 \%$ of them were Ryukyuan, suggesting that genetic background would not play a part in the differences between the two groups in this study. In particular, A. muciniphila colonizes the human gut within a year of birth [22], suggesting that the area where a person lives in early life contributes to the differences in colonization rate of this bacteria. We concluded that the differences in A. muciniphila colonization rate between groups OG and NOG may have been due to whether the participants lived in Ogimi during the first few years of their lives. We attempted to determine the factors affecting the gut microbiota in our participants, but the living environment, lifestyle, and food in the village have changed in the past 70 to 90 years. Therefore, the reasons behind the high rate of $A$. muciniphila colonization in the women who lived in Ogimi in early life are unclear.

In a Chinese study, the relative abundance of Akkermansia was significantly lower in a centenarian group than in an 85 to 99 years old group [16]. In contrast, an Italian study and a South Korean study both showed that the abundance of Akkermansia was higher in a centenarian group than in an elderly group [15, 17]. Whether Akkermansia is related to longevity is unclear; however, it is notable that the elderly women who were born in Ogimi have a higher rate of $A$. muciniphila colonization than other women. A follow-up study is needed to determine whether our participants who had A. muciniphila in their guts live longer.

\section{DATA AVAILABILITY}

16S rRNA gene sequence data have been deposited into the DNA Data Bank of Japan (DDBJ) under the accession no. DRA010457.

\section{ACKNOWLEDGEMENT}

We would like to thank Y. Sunagawa, R. Minei, S. Sekaryo, C. Uehara, N. Suzuki, and K. Chinen (National Institute of Technology, Okinawa College) for communicating with the participants and instructing them on how to collect fecal samples.

\section{REFERENCES}

1. Shao Y, Forster SC, Tsaliki E, Vervier K, Strang A, Simpson N, Kumar N, Stares MD, Rodger A, Brocklehurst P, Field N, Lawley TD. 2019. Stunted microbiota and opportunistic pathogen colonization in caesarean-section birth. Nature 574: 117-121. [Medline] [CrossRef]

2. Lee SA, Lim JY, Kim BS, Cho SJ, Kim NY, Kim OB, Kim Y. 2015. Comparison of the gut microbiota profile in breast-fed and formula-fed Korean infants using pyrosequencing. Nutr Res Pract 9: 242-248. [Medline] [CrossRef]

3. Thompson AL, Monteagudo-Mera A, Cadenas MB, Lampl ML, Azcarate-Peril MA. 2015. Milk- and solid-feeding practices and daycare attendance are associated with differences in bacterial diversity, predominant communities, and metabolic and immune function of the infant gut microbiome. Front Cell Infect Microbiol 5: 3. [Medline] [CrossRef]

4. Azad MB, Konya T, Maughan H, Guttman DS, Field CJ, Chari RS, Sears MR, Becker AB, Scott JA, Kozyrskyj AL, CHILD Study Investigators 2013. Gut microbiota of healthy Canadian infants: profiles by mode of delivery and infant diet at 4 months. CMAJ 185: 385-394. [Medline] [CrossRef]

5. Rodríguez JM, Murphy K, Stanton C, Ross RP, Kober OI, Juge N, Avershina E, Rudi K, Narbad A, Jenmalm MC, Marchesi JR, Collado MC. 2015. The composition of the gut microbiota throughout life, with an emphasis on early life. Microb Ecol Health Dis 26: 26050. [Medline]

6. Kashtanova DA, Popenko AS, Tkacheva ON, Tyakht AB, Alexeev DG, Boytsov SA. 2016. Association between the gut microbiota and diet: Fetal life, early childhood, and further life. Nutrition 32: 620-627. [Medline] [CrossRef]

7. Koenig JE, Spor A, Scalfone N, Fricker AD, Stombaugh J, Knight R, Angenent LT, Ley RE. 2011. Succession of microbial consortia in the developing infant gut microbiome. Proc Natl Acad Sci USA 108 Suppl 1: 4578-4585. [Medline] [CrossRef]

8. Nishijima S, Suda W, Oshima K, Kim SW, Hirose Y, Morita H, Hattori M. 2016. The gut microbiome of healthy Japanese and its microbial and functional uniqueness. DNA Res 23: 125-133. [Medline] [CrossRef]

9. Mariat D, Firmesse O, Levenez F, Guimarăes V, Sokol H, Doré J, Corthier G, Furet JP. 2009. The Firmicutes/Bacteroidetes ratio of the human microbiota changes with age. BMC Microbiol 9: 123. [Medline] [CrossRef]

10. Biagi E, Nylund L, Candela M, Ostan R, Bucci L, Pini E, Nikkila J, Monti D, Satokari R, Franceschi C, Brigidi P, De Vos W. 2010. Through ageing, and beyond: gut microbiota and inflammatory status in seniors and centenarians. PLoS One 5: e10667. [Medline] [CrossRef]

11. Jinam T, Nishida N, Hirai M, Kawamura S, Oota H, Umetsu K, Kimura R, Ohashi J, Tajima A, Yamamoto T, Tanabe H, Mano S, Suto Y, Kaname T, Naritomi K, Yanagi K, Niikawa N, Omoto K, Tokunaga K, Saitou N, Japanese Archipelago Human Population Genetics Consortium 2012. The history of human populations in the Japanese Archipelago inferred from genome-wide SNP data with a special reference to the Ainu and the Ryukyuan populations. J Hum Genet 57: 787-795. [Medline] [CrossRef]

12. Taira K, Tanaka H, Arakawa M, Nagahama N, Uza M, Shirakawa S. 2002. Sleep health and lifestyle of elderly people in Ogimi, a village of longevity. Psychiatry Clin Neurosci 56: 243-244. [Medline] [CrossRef]

13. Cox PA, Metcalf JS. 2017. Traditional food items in Ogimi, Okinawa: 1-Serine content and the potential for neuroprotection. Curr Nutr Rep 6: 24-31. [Medline] [CrossRef]

14. Takimoto T, Hatanaka M, Hoshino T, Takara T, Tanaka K, Shimizu A, Morita H, Nakamura T. 2018. Effect of Bacillus subtilis C-3102 on bone mineral density in healthy postmenopausal Japanese women: a randomized, placebo-controlled, doubleblind clinical trial. Biosci Microbiota Food Health 37: 87-96. [Medline] [CrossRef]

15. Biagi E, Franceschi C, Rampelli S, Severgnini M, Ostan R, Turroni S, Consolandi C, Quercia S, Scurti M, Monti D, Capri M, Brigidi P, Candela M. 2016. Gut microbiota and extreme longevity. Curr Biol 26: 1480-1485. [Medline] [CrossRef]

16. Wang F, Yu T, Huang G, Cai D, Liang X, Su H, Zhu Z, Li D, Yang Y, Shen P, Mao R, Yu L, Zhao M, Li Q. 2015. Gut microbiota community and its assembly associated with age and diet in Chinese centenarians. J Microbiol Biotechnol 25: 1195-1204. [Medline] [CrossRef]

17. Kim BS, Choi CW, Shin H, Jin SP, Bae JS, Han M, Seo EY, Chun J, Chung JH 2019. Comparison of the gut microbiota of centenarians in longevity villages of South Korea with those of other age groups. J Microbiol Biotechnol 29: 429-440. [Medline] 
[CrossRef]

18. Naito Y, Takagi T, Inoue R, Kashiwagi S, Mizushima K, Tsuchiya S, Itoh Y, Okuda K, Tsujimoto Y, Adachi A, Maruyama N, Oda Y, Matoba S. 2019. Gut microbiota differences in elderly subjects between rural city Kyotango and urban city Kyoto: an age-gender-matched study. J Clin Biochem Nutr 65: 125-131. [Medline] [CrossRef]

19. Derrien M, Vaughan EE, Plugge CM, de Vos WM. 2004. Akkermansia muciniphila gen. nov., sp. nov., a human intestinal mucin-degrading bacterium. Int J Syst Evol Microbiol 54: 1469-1476. [Medline] [CrossRef]

20. Everard A, Belzer C, Geurts L, Ouwerkerk JP, Druart C, Bindels LB, Guiot Y, Derrien M, Muccioli GG, Delzenne NM, de Vos WM, Cani PD. 2013. Cross-talk between Akkermansia muciniphila and intestinal epithelium controls diet-induced obesity. Proc Natl Acad Sci USA 110: 9066-9071. [Medline] [CrossRef]

21. Depommier C, Everard A, Druart C, Plovier H, Van Hul M, Vieira-Silva S, Falony G, Raes J, Maiter D, Delzenne NM, de Barsy M, Loumaye A, Hermans MP, Thissen JP, de Vos WM, Cani PD. 2019. Supplementation with Akkermansia muciniphila in overweight and obese human volunteers: a proof-of-concept exploratory study. Nat Med 25: 1096-1103. [Medline] [CrossRef]

22. Collado MC, Derrien M, Isolauri E, de Vos WM, Salminen S. 2007. Intestinal integrity and Akkermansia muciniphila, a mucin-degrading member of the intestinal microbiota present in infants, adults, and the elderly. Appl Environ Microbiol 73: 7767-7770. [Medline] [CrossRef]

23. Odamaki T, Kato K, Sugahara H, Hashikura N, Takahashi S, Xiao JZ, Abe F, Osawa R. 2016. Age-related changes in gut microbiota composition from newborn to centenarian: a cross-sectional study. BMC Microbiol 16: 90. [Medline] [CrossRef]

24. Deschasaux M, Bouter KE, Prodan A, Levin E, Groen AK, Herrema H, Tremaroli V, Bakker GJ, Attaye I, Pinto-Sietsma SJ, van Raalte DH, Snijder MB, Nicolaou M, Peters R, Zwinderman AH, Bäckhed F, Nieuwdorp M. 2018. Depicting the composition of gut microbiota in a population with varied ethnic origins but shared geography. Nat Med 24: 1526-1531. [Medline] [CrossRef] 